\title{
Confined Tamm optical states coupled to quantum dots in a photoconductive detector
}

Cite as: Appl. Phys. Lett. 115, 171101 (2019); https://doi.org/10.1063/1.5121597

Submitted: 26 July 2019 . Accepted: 09 October 2019 . Published Online: 21 October 2019

Edmund Harbord, Ben Cemlyn (D), Matthew Parker, Edmund Clarke (iD), Kenneth Kennedy, lan Henning, Mike Adams, and Ruth Oulton
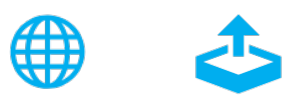

\section{ARTICLES YOU MAY BE INTERESTED IN}

High power surface emitting InGaN superluminescent light-emitting diodes

Applied Physics Letters 115, 171102 (2019); https://doi.org/10.1063/1.5118953

Optimization of the squeezing factor by temperature-dependent phase shift compensation in a doubly resonant optical parametric oscillator

Applied Physics Letters 115, 171103 (2019); https://doi.org/10.1063/1.5115795

Dielectric nanoresonators and metamaterials

Journal of Applied Physics 126, 150401 (2019); https://doi.org/10.1063/1.5129100

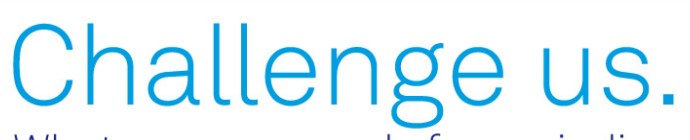

What are your needs for periodic signal detection?

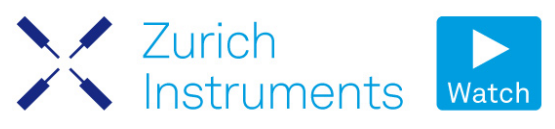

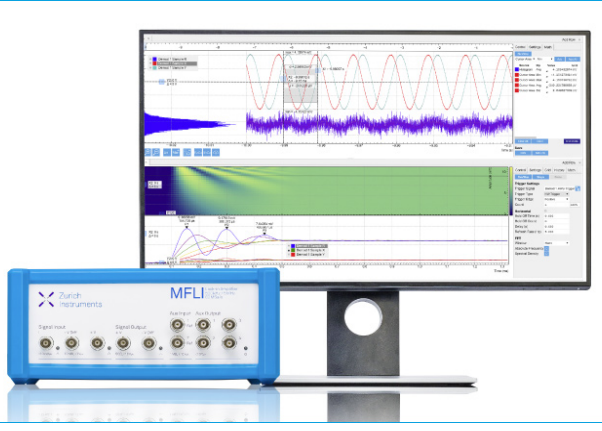

115, 171101 


\title{
Confined Tamm optical states coupled to quantum dots in a photoconductive detector
}

\author{
Cite as: Appl. Phys. Lett. 115, 171101 (2019); doi: 10.1063/1.5121597 \\ Submitted: 26 July 2019 - Accepted: 9 October 2019 . \\ Published Online: 21 October 2019
}

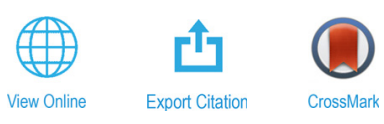

Edmund Harbord, ${ }^{1, a)}$ Ben Cemlyn, ${ }^{2}$ (D) Matthew Parker, Edmund Clarke, ${ }^{3}$ (D) Kenneth Kennedy, ${ }^{3}$ lan Henning, ${ }^{2}$ Mike Adams, ${ }^{2}$ and Ruth Oulton

\begin{abstract}
AFFILIATIONS
${ }^{7}$ Quantum Engineering Technology Labs, School of Physics and Department of Electrical and Electronic Engineering, University of Bristol, 5 Tyndall Ave., Bristol BS8 1FD, United Kingdom

${ }^{2}$ School of Computer Science and Electronic Engineering, University of Essex, Wivenhoe Park, Colchester CO4 3SQ, United Kingdom ${ }^{3}$ EPSRC National Epitaxy Facility, Centre for Nanoscience and Technology, University of Sheffield, North Campus, Broad Lane, Sheffield S3 7HQ, United Kingdom

a) Author to whom correspondence should be addressed: edmund.harbord@gmail.com
\end{abstract}

\begin{abstract}
By employing Tamm optical states-states localized between a thin metal microdisk and a semiconductor distributed Bragg reflector-we demonstrate near $1300 \mathrm{~nm}$ (O-band), narrow wavelength (20 nm FWHM), highly spatially localized photodetection. By varying the size of the microdisk, we show spectral tuning $(7 \mathrm{~nm})$ of the peak optical response. Furthermore, by reducing the symmetry of the microstructures, we lift the degeneracy of the polarization, producing a polarization sensitivity in our detector. These confined Tamm states are useful not only for sensing but also for photoconductive switch applications such as optical microwave switches and terahertz generation.
\end{abstract}

Published under license by AIP Publishing. https://doi.org/10.1063/1.5121597

In the early days of solid state physics, Igor Tamm proposed ${ }^{1}$ that localized electronic states (Tamm states) would be found at the surface of an insulator, arising from the termination of a regularly spaced crystal lattice. The optical analog of these arises by the localization of the light field due to the termination of a regularly spaced photonic crystal lattice-Tamm optical states. One class of Tamm optical states occurs when the photonic crystal is terminated by a metallic layer, with an imaginary component of the refractive index. These states were proposed ${ }^{2}$ and observed, ${ }^{3}$ being formed between a metal layer and a distributed Bragg reflector (DBR). These Tamm states are topologically protected ${ }^{4}$ and are readily formed by the deposition of a single, thin, metallic layer on top of a DBR. The simplicity of fabrication, compared to, for example, etching DBR cavity structures, has attracted a great deal of interest for sensing, ${ }^{5,6}$ including temperature detection ${ }^{7}$ and enhanced hot-electron photodetection. ${ }^{8}$ By fabricating microstructures a few micrometers in size, it is possible to confine the light in all three dimensions. ${ }^{9}$ This has been used to demonstrate several useful devices, including a quantum dot (QD) based single photon source ${ }^{10}$ and nanolasers, ${ }^{11}$ as well as designs for solar cells ${ }^{12}$ and photodetectors. ${ }^{13}$ It is important to distinguish Tamm states from conventional surface plasmons. In the case of conventional states, the boundary condition requires that the electric field is maximized at the metal/dielectric interface, leading to substantial propagation losses in the metal. By contrast, in Tamm states, the electric field maximum lies within the dielectric spacer layer, reducing these losses.

In this letter, we demonstrate a Tamm state photodetector operating in the $\mathrm{O}$ band. By using confined Tamm states of different sizes, we can tune the spectral response of the photodetector, and by using low symmetry structures, we also obtain a polarization sensitive response. We suggest our device is useful not only for multispectral measurements but also for photoconductive switch applications such as terahertz ${ }^{14}$ generation and microwave optical switching. ${ }^{15}$

A cross section of a basic layer structure designed to produce Tamm modes is shown in Fig. 1(a). This incorporates a distributed Bragg reflector, centered nominally at $1300 \mathrm{~nm}$, on which there is a thin $(75 \mathrm{~nm})$ spacer layer of GaAs and a thin $(25 \mathrm{~nm})$ layer of gold. This design was previously shown to form a Tamm mode at $1300 \mathrm{~nm}^{16}$ The reflectivity of such structures is calculated by the 1D transfer matrix method (TMM) ${ }^{17}$ and shown in Fig. 1(b), for the bare DBR and spacer layer (blue) and the gold-capped structure (yellow). The pronounced dip visible in the gold-capped structure is the Tamm mode at $1300 \mathrm{~nm}$. The electric field profile within the device as calculated at $1300 \mathrm{~nm}$ is shown in Fig. 1(c), together with the refractive 

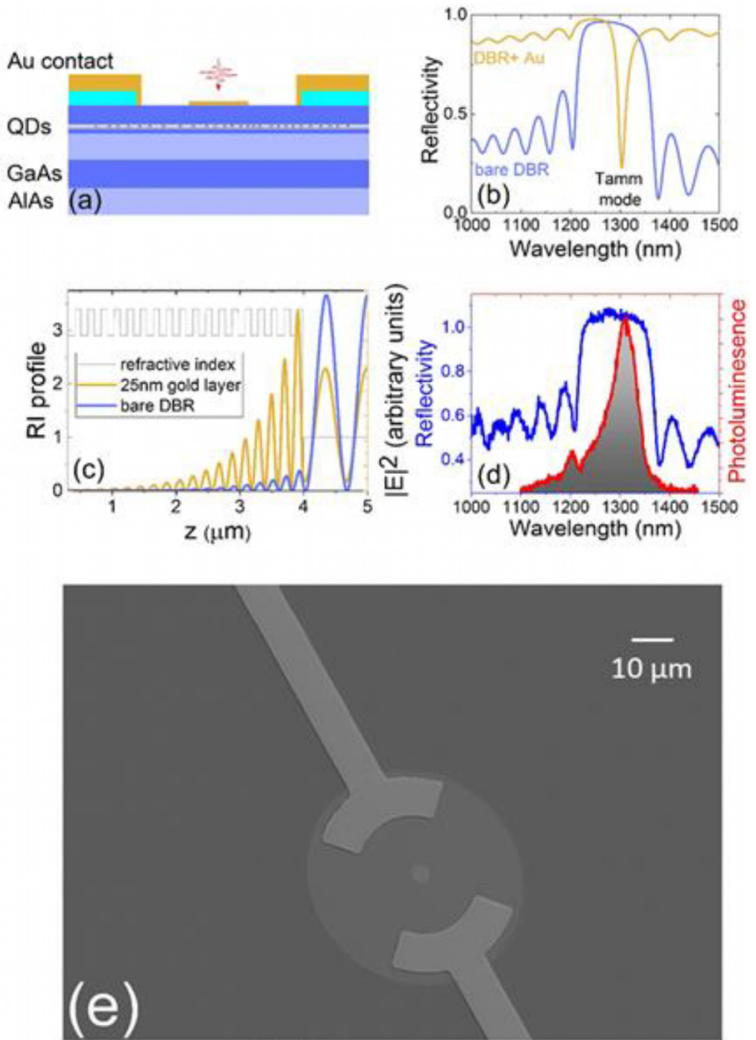

FIG. 1. (a) Schematic of a cross section of a photoconductive device incorporating a Tamm structure, consisting of a GaAs/AlAs distributed Bragg reflector with a thin layer of gold on the top to provide the optical confinement. (b) Calculated reflectivity by the $1 \mathrm{D}$ transfer matrix method for the reflectivity of a 17.5 pair DBR with a $75 \mathrm{~nm}$ GaAs spacer layer (blue) and with a $25 \mathrm{~nm}$ layer of gold on top (yellow). (c) The electric field calculated for the bare DBR (blue) and the Tamm mode (yellow). (d) Characterization of the as-grown sample: photoluminescence (red, with gray shade) and reflectivity (blue). (e) Electron microscopy image showing the fabricated device, with the Tamm disk in the middle and $\mathrm{C}$-shaped contacts on either side to allow the photoresistance to be measured.

index profile. Without the gold layer (blue), the light barely penetrates the upper layers of the device; however, with the gold layer (yellow), the field is strongly enhanced, especially in the spacer layer. This enhances the light-matter interaction underneath the gold layer, the geometry and dimension of which can be used to define the Tamm state.

In this work, we use InAs/GaAs quantum dots (QDs) as our active material for light absorption and detection. QDs-nanoscale inclusions of one semiconductor embedded in another-confine electrons and holes in all 3 dimensions to form discrete, atomiclike electronic states. They offer high internal quantum efficiency (approaching unity at cryogenic temperatures), ${ }^{18}$ and QDs of different sizes have different degrees of optical confinement and so couple to different wavelengths of light, making them ideal active materials for a photodetector with a broad spectral response. As a proof of concept, we adapt the well-known and readily fabricated photoresistive detector architecture, described below.

The wafer is grown by solid-source molecular beam epitaxy on a semi-insulating GaAs substrate. The layers consist of a Bragg reflector with 17.5 pairs of AlAs/GaAs (of thickness $94.5 \mathrm{~nm} / 110.8 \mathrm{~nm}$, respectively) followed by a GaAs spacer layer of $75 \mathrm{~nm}$. This is designed to give a Tamm mode at $1300 \mathrm{~nm}$ after the deposition of $25 \mathrm{~nm}$ of gold. A layer of QDs incorporated into a $5 \mathrm{~nm} \operatorname{In}_{0.18} \mathrm{Ga}_{0.82} \mathrm{As}$ quantum well $^{19-21}$ is positioned at $4 \mathrm{~nm}$ from the top AlAs/GaAs interface, simulations of which ${ }^{16}$ indicate that it is close to the peak of the electric field within this structure. Atomic force microscopy measurements of uncapped QDs grown under the same conditions indicate a dot density of $5.8 \times 10^{10} \mathrm{~cm}^{-2}$. The upper $50 \mathrm{~nm}$ of the spacer layer is $\mathrm{n}$-doped with silicon at a dopant density of $2 \times 10^{18} \mathrm{~cm}^{-3}$ in order to readily form low resistance electrical contacts.

Characterization of the as-grown wafer (without the metal layer) is shown in Fig. 1(d). The broadband reflectivity, shown in blue, agrees well with the TMM prediction for this design. Photoluminescence (with excitation above the GaAs band edge at $650 \mathrm{~nm}$ ) shows bright, spectrally broad QD emission centered near $1300 \mathrm{~nm}$ with a FWHM of $55 \mathrm{~nm}$. This corresponds to a wide range of QD sizes, which is more suited for broadband detection. A lesser peak in the emission at around $1200 \mathrm{~nm}$ is attributed to a side peak in the DBR response, which can also be seen in the reflectivity spectrum. We were unable to observe any PL with the gold layer deposited.

Confined Tamm modes are formed by patterning the metal layer laterally; the states can only exist under the metal, and so the lateral confinement creates an optical state confined in all 3 dimensions, analogous to those of a conventional waveguide. This increased confinement is shown to blueshift the mode. , 10,22,23 $^{2}$ Therefore, by changing the size of the micrometer scale structures, the confinement changes, and the mode can be spectrally tuned. By breaking the symmetry of the structures, the degeneracy of the polarization of the mode can be lifted and control the polarization of the light it couples to.

For ease of fabrication and potentially high device yields, a very simple fabrication process was chosen using one stage of photolithography with metal lift-off to define the metal Tamm disks and contacts. Moreover, comparatively large device dimensions, which have not been optimized for high performance, were selected for ease of definition with optical photolithography. The Tamm modes form under an illuminated gold pad, which are located between gold contacts to the surface $\mathrm{n}-\mathrm{GaAs}$ layer. Light absorbed here is seen as a photocurrent/ variation in conductivity between the contacts. A SEM image of a completed device is shown in Fig. 1(e); the two C-shaped n-type contacts are connected to two large bond-pads, which were used to apply bias and for the photocurrent measurement.

The experimental setup shown in Fig. 2 has been designed to explore the optical response of the Tamm photoconductor. Our illumination system consisted of a high NA (approximately 0.2, spot size $2.5 \mu \mathrm{m}$ FWHM) lens-ended fiber that was aligned with the center of the gold disks, thereby achieving stability and constant illumination properties. A widely tunable laser source provided narrowband illumination of the devices at a power of $0.6 \mathrm{~mW}$. A polarization controller was included, and a circulator enabled light reflected back from the device (and coupled into the fiber) to be viewed on an optical spectrum analyzer (OSA). The optical coupling into the Tamm structure was optimized by monitoring the OSA power using CW illumination: the returned power showed a clear minimum when the fiber was accurately centered on the disk, indicating that the resonant light was being coupled efficiently into the Tamm mode cf. the cavity resonance in Fig. 1(b). 


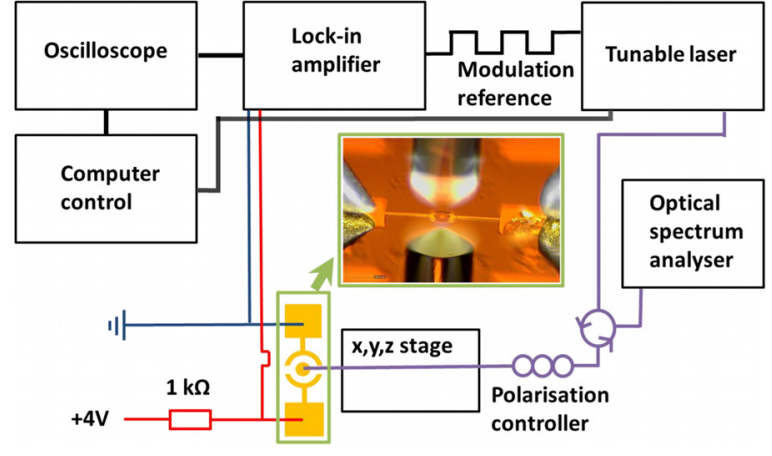

FIG. 2. Experimental setup, with the image of the fiber alignment and electrical contacts. The fiber is butted onto the disk when measurements are taken.

Due to the single-deposition fabrication process used here to form both the Tamm structures and contacts, the resulting devices used Schottky contacts, which reduced the DC response of the devices (i.e., under $\mathrm{CW}$ illumination).

We therefore used a modulated optical signal, together with a lock-in amplifier. Using this approach, a good Tamm response was achieved with modulation frequencies from $150 \mathrm{~Hz}$ up to $30 \mathrm{kHz}$, with the high frequency response ultimately restricted by the electrical set up. A "standard" modulation frequency of $1 \mathrm{kHz}$ was used to test a variety of disk sizes, which ranged from $7 \mu \mathrm{m}$ up to $20 \mu \mathrm{m}$.

We initially used a broad wavelength scan from $1270 \mathrm{~nm}$ to $1330 \mathrm{~nm}$ on a $13 \mu \mathrm{m}$ diameter disk to reveal the general spectral photoconductive response of the device. The light was of arbitrary polarization state. This is shown in Figs. 3(a) and 3(b). When focusing on the Tamm disk [Fig. 3(a), red line], a peak in the photocurrent response with a full width at half maximum of $\sim 20 \mathrm{~nm}$, centered near $1300 \mathrm{~nm}$, was observed. The response is asymmetric in the tails, falling off more rapidly on the short wavelength side compared to the long wavelength side, but it clearly shows that the Tamm mode is blueshifted from, and much narrower than, the QD PL spectrum. Figure 3(b) illustrates this further by showing the difference in the optical response when illuminating on the gold disk (Tamm response) compared to the regions between the $\mathrm{C}$ contact and the disk (background QD response). Taken together, these results provide clear association of the on-disk response with the Tamm modes, rather than simple absorption in the underlying QD layer.
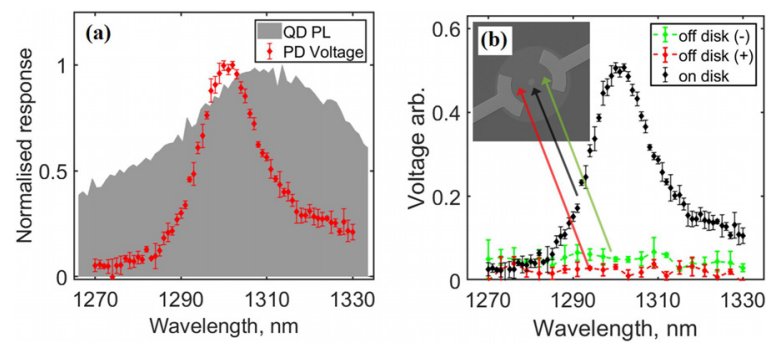

FIG. 3. Wide wavelength-scan response of a typical disk (a) scan in red overlaying QD PL profile in gray and (b) scan in black along with response off disk, toward the + - electrical contact sides.
Measurements were made over a frequency range of $1270 \mathrm{~nm}-$ $1310 \mathrm{~nm}$, where the photoresponse could be fitted to a Gaussian curve to derive the spectral position of the Tamm resonance peak in a consistent way. We found a distribution of resonance peaks between 1295 and $1305 \mathrm{~nm}$ and a blueshifting trend with a reduced disk size. In general, this accords well with previously published theoretical results for both this structure ${ }^{16}$ and a similar one operating in the $880-910 \mathrm{~nm}$ region. ${ }^{9}$ In Fig. 4(a), we plot the computed wavelength values of the circularly symmetric linearly polarized $\mathrm{LP}_{0 \mathrm{~m}}$ modes ${ }^{24}$ as a function of disk size. This plot was obtained using a novel approach based on the effective index method ${ }^{22,25}$ to determine the index profile of the circular cylindrical waveguide, as well as a hard mirror approximation for the cavity. ${ }^{26}$ Full details of this approach, which offers more physical insight into the analysis of confined Tamm modes, are given in our recent paper. $^{22}$

While the smallest disk sizes tend toward single mode operation, the larger devices are predicted to support a number of spatial modes. Accordingly, the spectral response arises from the convolution of the spectral mode with the QD distribution (which is much broader than the individual separation between the confined modes) and from the coupling of the incoming mode into several spatial modes. However, coupling into each of these modes depends additionally on the spatial overlap between the excitation mode and the particular mode of the disk under consideration. We illustrate this in Fig. 4(b). We consider only $\mathrm{LP}_{0 \mathrm{~m}}$ modes on symmetry grounds-the overlap integral between orthogonal modes will be small.

For the smallest disks [Fig. 4(b-i)], we can see that there is good overlap between the $\mathrm{LP}_{01}$ mode and the fiber mode, but as the disk gets larger [Fig. 4(b-ii)], this overlap markedly decreases. For the $\mathrm{LP}_{03}$ mode of the $7 \mu \mathrm{m}$ disk, there is also a good overlap [Fig. 4(b-iii)] with the incoming spot, supporting the resonances in Fig. 4(a), which appear to distinguish both $\mathrm{LP}_{01}$ and $\mathrm{LP}_{03}$ modes for the $7 \mu \mathrm{m}$ disk. However, for larger disks [Figs. 4(b-iv) and 4(b-v)], the overlap is better for higher order modes $\left(\mathrm{LP}_{03}, \mathrm{LP}_{06}\right)$. As these modes become spectrally closer together for very large disks, it would become impossible to distinguish between the modes. To optimize the spectral response, a thicker metal layer would increase the separation between modes and improve the spectral dependence of the response. In addition, by using several adjacent disks between the C-shaped contacts, we can minimize the spatial dependence of the photodetector. The apparent shifts in resonance on repeated tests of a disk, due to excitation of different lateral modes, can be understood as due to different launch conditions. Factors such as the focused spot size in the QD region, surface irregularities, and contamination may change the excitation profile in the material sufficiently to excite different modes. Exemplary scans are shown in Fig. 5 for the $11 \mu \mathrm{m}$ and $15 \mu \mathrm{m}$ disks. Previous measurements by Fourier microscopy on confined Tamm structures have indicated ${ }^{27,28}$ that excitation of large Tamm disks at different real-space positions results in different spectral responses in $\mathrm{k}$-space. This behavior is the real-space equivalent of this $\mathrm{k}$-space behavior.

The blueshift with the reducing disk dimension suggests that breaking the symmetry of the gold microstructures might lead to polarization discrimination (polarized modes for realizing low temperature nanolasers have been demonstrated using this effect ${ }^{29}$ ). To verify this, we calibrated the polarization of our illumination system using a collimating lens and free space polarimeter behind the sample. Once 
(a)

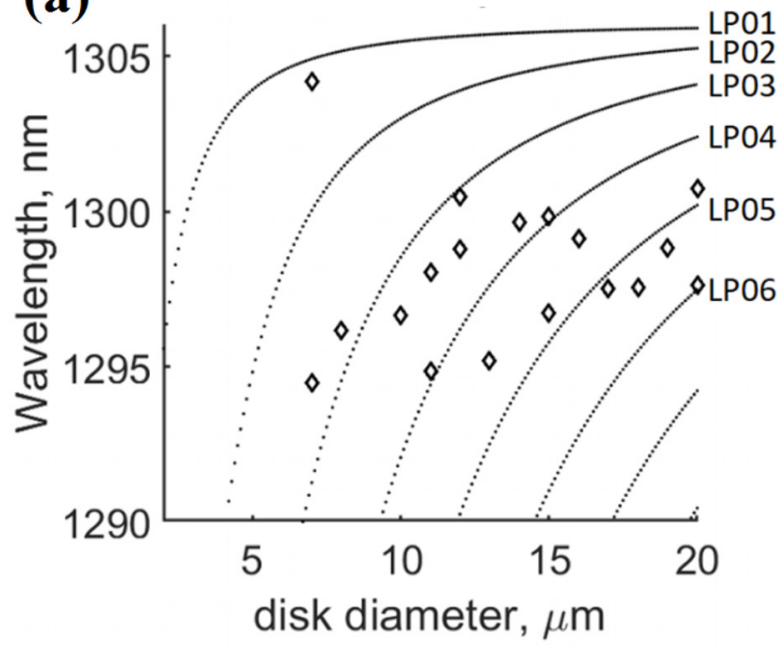

(b)
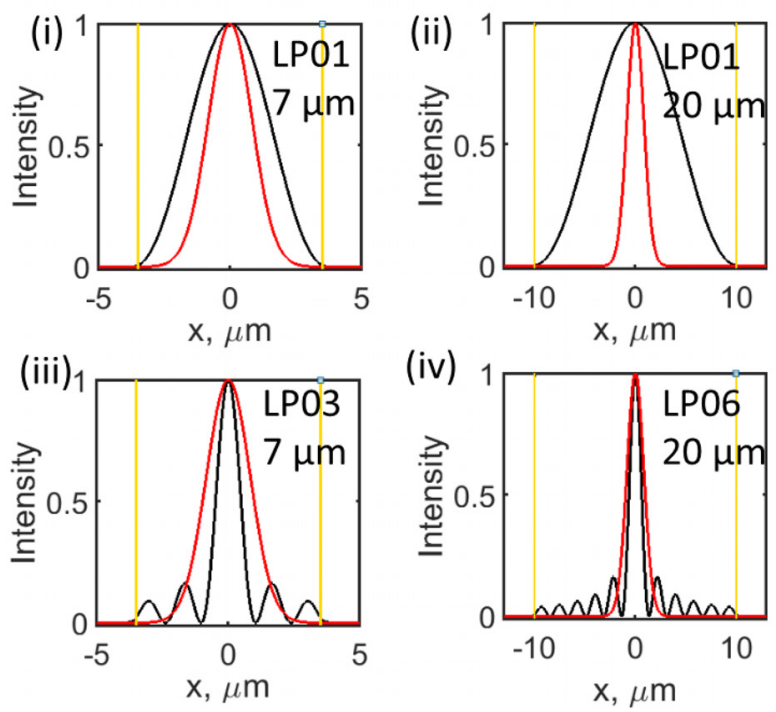
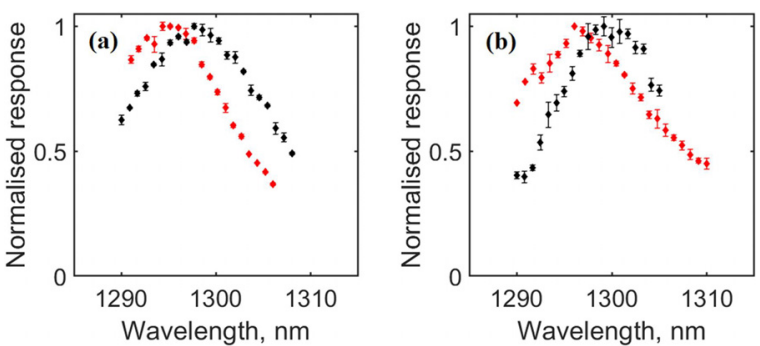

FIG. 5. Repeated scans with different alignments for the larger disks (a) $11 \mu \mathrm{m}$ and (b) $15 \mu \mathrm{m}$. This reflects the sensitivity of disks to the launch conditions as anticipated due to the multimode structure.

calibrated, the polarization was easily varied with the polarization controller indicated in Fig. 2, without disturbing the alignment of the fiber. Instead of circular disks, we tested an asymmetric, $3 \mu \mathrm{m} \times 8 \mu \mathrm{m}$ rectangular device [Fig. 6(a)] under illumination with light of $\mathrm{H}(\mathrm{V})$ polarization selectively aligned with the long (short) axis, respectively.

Here, the device was excited with the fiber positioned approximately 2-3 $\mu \mathrm{m}$ from the surface. Figure 6 (b) shows the photoresponse as a function of wavelength taken for each polarization, showing a relative blueshift for $\mathrm{V}$ polarized light. This will be subject to the spatial overlaps of the allowed $\mathrm{H} / \mathrm{V}$ modes and the illumination profile; nevertheless, the result does suggest that improved polarization discrimination might be realized in an optimized device.

We discuss briefly how to optimize such a polarization response: by using the metal thickness and/or the spacer thickness to red-detune the mode from the QD peak photoresponse and increasing the asymmetry of the device, ${ }^{30-32}$ we can enhance the differential response between the two orthogonal polarizations. Coupling via a Gaussian spot would be improved by using interdigital electrodes over the detection area. A complementary approach for a polarization sensitive device would be to use a spectrally narrower detecting region such as a quantum well or an annealed ${ }^{33,34}$ QD population, which would increase further this differential response.

In conclusion, we have demonstrated wavelength and polarization selective detection using the confined Tamm modes within a photoconductive photodetector. Our results on devices of varying the size and geometry suggest that this could offer a promising design route for device optimization in terms of wavelength and polarization (a)

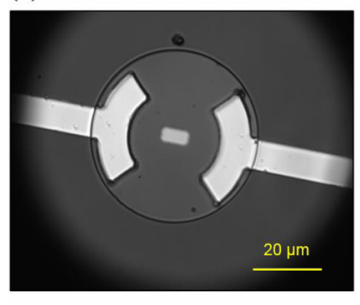

(b)

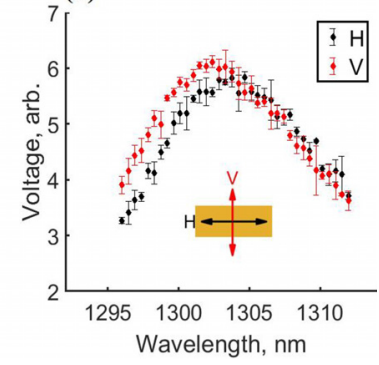

FIG. 4. Tamm responses for different disk sizes. (a) Peak resonances from Gaussian fittings for all disk sizes, together with calculated $\mathrm{LP}_{0 \mathrm{~m}}$ modes (see the text). (b) Comparison of the fiber mode spatial profile at the disk (red) with the calculated mode (black) for a given diameter of disk, with the boundary of the disk illustrated by the yellow lines. The modes shown are $\mathrm{LP}_{01}$ for (i) $7 \mu \mathrm{m}$ and (ii) 20 $\mu \mathrm{m}$ disks; (iii) $\mathrm{LP}_{03}$ mode for a $7 \mu \mathrm{m}$ disk; (iv) the $\mathrm{LP}_{06}$ mode for a $20 \mu \mathrm{m}$ disk; and (v) the $\mathrm{LP}_{03}$ mode for a $12 \mu \mathrm{m}$ disk.

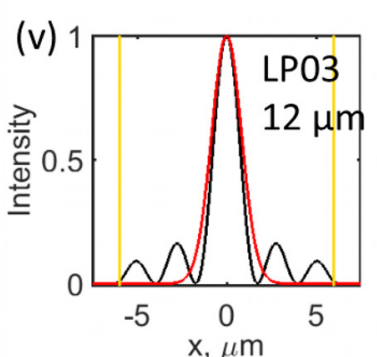
of the device with linear polarized light parallel to $(H)$ and perpendicular to $(V)$ the longest dimension. 
response and optical coupling efficiency. Moreover, the small size of confined Tamm devices renders them suitable for integrating into a pixel-based spectral and polarization-resolved detector. These could prove ideal for chip-level sensing, with a robust and simple-to-fabricate structure. While these Tamm mode photodetectors based on telecom wavelength semiconductor QDs offer a simple way to fabricate cavity-enhanced photodetectors with a single, thin metal layer (instead of a full upper Bragg stack), there are possible applications beyond this, for example, in the enhancement of photoconductive switching. Photoconductive switches have key applications including the generation of terahertz radiation ${ }^{14}$ and optical microwave switching. ${ }^{15}$

We acknowledge support from EPSRC under project SPIN SPACE (Grant Nos. EP/M024156/1 and EP/M024237/1) and No. 1D QED EP/N003381/1. We gratefully acknowledge two anonymous reviewers for their comments on our paper.

\section{REFERENCES}

${ }^{1}$ I. Tamm, Phys. Z. Sov. Union 1, 733 (1932).

${ }^{2}$ M. Kaliteevski, I. Iorsh, S. Brand, R. A. Abram, J. M. Chamberlain, A. V. Kavokin, and I. A. Shelykh, Phys. Rev. B 76, 165415 (2007).

${ }^{3}$ M. E. Sasin, R. P. Seisyan, M. A. Kaliteevski, S. Brand, R. A. Abram, J. M. Chamberlain, A. Yu. Egorov, A. P. Vasil'ev, V. S. Mikhrin, and A. V. Kavokin, Appl. Phys. Lett. 92, 251112 (2008).

${ }^{4}$ M. Xiao, Z. Q. Zhang, and C. T. Chan, Phys. Rev. X 4, 021017 (2014).

${ }^{5}$ B. Auguie, M. C. Fuertes, P. C. Angelomé, N. Lo'pez Abdala, G. J. A. A. Soler Illia, and A. Fainstein, ACS Photonics 1, 775 (2014).

${ }^{6}$ W. L. Zhang, F. Wang, Y. J. Rao, and Y. Jiang, Opt. Express 22, 14524 (2014).

${ }^{7}$ Y. Tsurimaki, J. K. Tong, V. N. Boriskin, A. Semenov, M. I. Ayzatsky, Y. P. Machekhin, G. Chen, and S. V. Boriskina, ACS Photonics 5, 929 (2018).

${ }^{8}$ C. Zhang, K. Wu, V. Giannini, and X. Li, ACS Nano 11, 1719 (2017).

${ }^{9}$ O. Gazzano, S. Michaelis de Vasconcellos, K. Gauthron, C. Symonds, J. Bloch, P. Voisin, J. Bellessa, A. Lemaitre, and P. Senellart, Phys. Rev. Lett. 107, 247402 (2011).

${ }^{10}$ O. Gazzano, S. Michaelis de Vasconcellos, K. Gauthron, C. Symonds, P. Voisin, J. Bellessa, A. Lemaitre, and P. Senellart, Appl. Phys. Lett. 100, 232111 (2012).

${ }^{11}$ C. Symonds, G. Lheureux, J. P. Hugonin, J. J. Greffet, J. Laverdant, G. Brucoli, A. Lemaitre, P. Senellart, and J. Bellessa, Nano Lett. 13, 3179 (2013).
${ }^{12}$ X. L. Zhang, J. F. Song, X. B. Li, J. Feng, and H. B. Sun, Appl. Phys. Lett. 101, 243901 (2012).

${ }^{13}$ K. Wu, Y. Zhan, C. Zhang, S. Wu, and X. Li, Sci. Rep. 5, 14304 (2015).

${ }^{14} \mathrm{E}$. Castro-Camus and M. Alfaro, Photonics Res. 4, A36 (2016).

${ }^{15}$ Y. Kaneko, T. Takenaka, T. S. Low, Y. Kondoh, D. E. Mars, D. Cook, and M. Saito, Electron. Lett. 39, 917 (2013).

${ }^{16}$ M. Parker, E. Harbord, A. Young, P. Androvitsaneas, J. Rarity, and R. Oulton, IEE Optoelectron. 12, 11 (2018).

${ }^{17}$ P. Yeh, J. Opt. Soc. Am. 69, 742 (1979).

${ }^{18}$ J. Johansen, S. Stobbe, I. S. Nikolaev, T. Lund-Hansen, P. T. Kristensen, J. M. Hvam, W. L. Vos, and P. Lodahl, Phys. Rev. B 77, 073303 (2008).

${ }^{19}$ K. Nishi, H. Saito, S. Sugou, and J.-S. Lee, Appl. Phys. Lett. 74, 1111 (1999).

${ }^{20}$ V. M. Ustinov, N. A. Maleev, A. E. Zhukov, A. R. Kovsh, A. Yu. Egorov, A. V. Lunev, B. V. Volovik, I. L. Krestnikov, Yu. G. Musikhin, N. A. Bert, P. S. Kop'ev, Zh. I. Alferov, N. N. Ledentsov, and D. Bimberg, Appl. Phys. Lett. 74, 2815 (1999)

${ }^{21}$ S. S. Alharthi, J. Orchard, E. Clarke, I. D. Henning, and M. J. Adams, Appl. Phys. Lett. 107, 151109 (2015).

${ }^{22}$ M. J. Adams, B. R. Cemlyn, I. D. Henning, M. Parker, E. Harbord, and R. Oulton, J. Opt. Soc. Am. B 36, 125 (2019).

${ }^{23}$ M. Parker, E. Harbord, L. Chen, E. Clarke, K. Kennedy, J. Rarity, and R. Oulton, "Telecommunication wavelength confined Tamm plasmon structures containing InAs/GaAs quantum dot emitters at room temperature," Phys. Rev. B (to be published); e-print arXiv:1903.01151v1.

${ }^{24} \mathrm{D}$. Gloge, Appl. Opt. 10, 2252-2258 (1971).

${ }^{25} \mathrm{G}$. R. Hadley, Opt. Lett. 20, 1483-1485 (1995).

${ }^{26}$ D. I. Babic and S. W. Corzine, IEEE J. Quantum Electron. 28, 514 (1992).

${ }^{27}$ F. Feng, C. Symonds, C. Schwob, J. Bellessa, A. Maitre, J.-P. Hugonin, and L. Coolen, New J. Phys. 20, 033020 (2018).

${ }^{28}$ F. Feng, W. Daney de Marcillac, X. Lafosse, S. L. Portalupi, M. Nasilowski, B. Dubertret, J.-M. Frigerio, C. Schwob, A. Maitre, P. Senellart, and L. Coolen, New J. Phys. 18, 083018 (2016).

${ }^{29}$ G. Lheureux, S. Azzini, C. Symonds, P. Senellart, A. Lemaitre, C. Sauvan, J.-P. Hugonin, J.-J. Greffet, and J. Bellessa, ACS Photonics 2, 842 (2015).

${ }^{30}$ M. J. Adams, D. N. Payne, and C. M. Ragdale, Electron. Lett. 15, 298 (1979).

${ }^{31}$ B. Gayral, J. M. Gerard, B. Legrand, E. Costard, and V. Thierry-Mieg, Appl. Phys. Lett. 72, 1421 (1998).

${ }^{32}$ R. Michalzik, M. Ostermann, and P. Debernardi, Proc. SPIE 6908, 69080A (2008).

${ }^{33}$ E. Harbord, P. Spencer, E. Clarke, and R. Murray, J. Appl. Phys. 105, 033507 (2009).

${ }^{34}$ E. Harbord, Y. Ota, Y. Igarashi, M. Shirane, N. Kumagai, S. Ohkouchi, S. Iwamoto, S. Yorozu, and Y. Arakawa, Jpn. J. Appl. Phys., Part 1 52, 125001 (2013). 